\title{
A rare presentation of tuberculous osteomyelitis in childhood
}

\author{
TMR Gunasekera $^{1}$, DH Karunathilake ${ }^{2}$, EMNW Wickramasinghe $^{3}$, KAHM Jayaweera $^{4}$ \\ Sri Lanka Journal of Child Health, 2008; 37: 61-2
}

(Key words: tuberculous osteomyelitis, children)

\section{Introduction}

Tuberculosis (TB) is a major health problem in the developing world and eight million new cases are detected annually with three million deaths ${ }^{1}$. Extra pulmonary manifestations of TB are very variable and lead to a diagnostic challenge. Tuberculous osteomyelitis accounts for $1-6 \%$ of extra pulmonary TB in childhood ${ }^{2}$. Axial skeleton is the commonest site of involvement in adults even though peripheral skeletal involvement is more common among children ${ }^{3}$. However, vertebrae are the most commonly affected bones in both groups ${ }^{4}$. Disseminated skeletal involvement is very rare in children and calvarial osteomyelitis is even rarer accounting for approximately $1 \%$ of skeletal tuberculous disease. We report a child with TB osteomyelitis who presented to us with multiple lytic lesions involving skull, ribs and long bones.

\section{Case report}

A nine year old girl presented to us with intermittent fever, generalized malaise, poor appetite and loss of weight over a period of three months and a limp involving the right hip of recent onset. No history of any respiratory tract problems, skin involvement or joint swellings was forthcoming during interrogation. She was born to non-consanguineous healthy parents and had been well until the onset of this illness. Other two siblings aged eleven and five remained well. There was no contact history of tuberculosis. She was born by elective caesarean section and had had no perinatal problems. Her development was age appropriate and she had received all EPI immunizations including BCG.

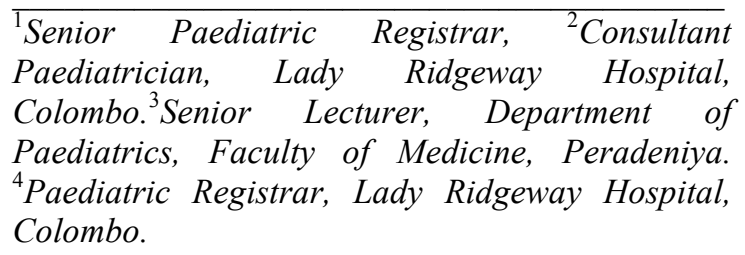

(Received on 21 March 2007. Accepted on 20 April 2007))
Examination revealed an acutely wasted child with significant pallor. BCG scar was present. There was no hepatoslenomegaly and examination of other systems was unremarkable. Right hip abduction and external rotation were limited, but other joint movements were full.

Investigations revealed a leucocyte count of $10 \times 10^{9} / \mathrm{L}$ with a normal differential count. Platelet count was $633 \times 10^{9} / \mathrm{L}$. Hemoglobin was $8.7 \mathrm{~g} / \mathrm{dl}$. Peripheral blood film showed evidence of anaemia of chronic disorder. ESR was $102 \mathrm{~mm}$ in first hour and the Mantoux response was $12 \mathrm{~mm}$ after 72 hours. Serum protein electrophoresis did not reveal any abnormal bands. An effusion in right hip joint measuring $1.6 \mathrm{~cm}$ with a periosteal reaction over the upper femur and lateral aspect of iliac bone on the same side was detected during ultrasound examination. Abdominal ultrasonography was normal. Skeletal $\mathrm{x}$-rays revealed multiple lytic lesions in ribs, pelvic bones and right femur. There were two punched out lesions in skull and a collapsed vertebra at mid thoracic level.

Sputum examination was negative for acid fast bacilli on three consecutive days. Bone marrow aspiration was unremarkable. Fungal blood cultures with special reference to histoplasmosis did not show any growth. Urinary Bence Jones proteins were negative and urinary VMA excretion was within normal range. The aspirate of right hip joint, done under ultrasound guidance yielded numerous pus cells and culture of the fluid revealed a growth of Mycobacterium tuberculosis. The bone biopsy from a lytic lesion in a rib revealed caseous necrosis with epitheloid granulomas and multiple giant cells.

She was commenced on anti TB medication as for disseminated bone TB and is being followed up as an outpatient.

\section{Discussion}

Osteomyelitis at multiple sites is a rare complication of childhood tuberculosis. It occurs due to lymphohaematogenous dissemination from 
lungs ${ }^{5}$, but the primary pulmonary lesion is not easily identifiable in children. The commonly affected sites in TB osteomyelitis are spine, femur, tibia and fibula in that order of frequency ${ }^{6}$. Infection starts in cancellous bony segments. In calvarial osteomyelitis, frontal and parietal skull bones are commonly involved due to the presence of large amounts of cancellous bone. Three types of skull lesions have been demonstrated roentgenographically, namely, perforating, diffuse and sclerotic forms ${ }^{7}$.

The anterior parts of vertebral bodies are affected initially in spinal TB. The resultant destruction leads to collapse and wedging of spine with gibbus formation. Paraspinal abscesses can cause cord compression leading to neurological deficits ${ }^{8}$.

TB osteomyelitis runs an insidious course. Localized pain and swelling are relatively common early clinical manifestations. Cutaneous stigmata such as chronic discharging sinuses are late manifestations. Constitutional symptoms are uncommon unless there is concomitant pulmonary disease 9 .

Diagnosis of TB osteomyelitis requires a high index of clinical suspicion. Markers of acute inflammation/infection such as ESR and $\mathrm{C}$ reactive proteins are elevated, but are nonspecific. Radiographic appearances of TB osteomyelitis depend on the stage of presentation at diagnosis, ranging from mild soft tissue swelling to areas of osteolysis with local osteopenia. Similar radiological findings may be seen in eosinophilic granuloma, disseminated lymphangiomatosis and in primary / secondary malignancy ${ }^{10}$ or in fungal infections such as histoplasmosis or cryptococosis. Collapsed vertebral bodies, narrowed intervening discs and large paravertebral abscesses may be visible on spinal $\mathrm{x}$ rays. CT, MRI or myelography may be more informative. Early morning gastric aspirates and joint effusion fluids rarely demonstrate acid fast bacilli in affected children but cultures for Mycobacterium tuberculosis are more informative with positive results in one third of cases ${ }^{11}$. This was the case in our patient. In those with negative cultures, definitive diagnosis rests on bone biopsy and demonstration of granuloma formation. Chest radiography is an unreliable tool even in adult patient population as only $15-20 \%$ of patients have concomitant active pulmonary disease ${ }^{6}$. Positive Mantoux reaction and polymerase chain reaction have improved the diagnostic yield, but exact sensitivity and specificity with regard to extra pulmonary $\mathrm{TB}$ are not yet known ${ }^{12}$.

The mainstay of treatment in tuberculous osteomyelitis is anti TB chemotherapy for a longer duration often as long as eighteen months ${ }^{13}$. Surgery may be indicated for large abscesses, spinal stabilization and when there is fulminating secondary bacterial infection.

\section{References}

1. Dolin PJ, Raviglione MC, Kochi A. Global tuberculosis incidence and mortality during 1990-2000. Bull World Health Organ 1994; 72:213-20.

2. Davidson PT, Horowitz I. Skeletal tuberculosis. Am J Med 1970; 48:77-84.

3. Vohra R, Kang HS, Dogra S, Saggar RR, Sharma R. Tuberculosis osteomyelitis. J Bone Joint Surg.1997; 79B:562-6.

4. Bavadekar AV. Osteoarticular tuberculosis in children. Prog Pediatr Surg 1982; 15:1131-51.

5. Huang CH. Extra-articular tuberculosis osteomyelitis. A report of 11 cases. Int Orthop 1996; 20:169-71.

6. Vallejo JG, Ong LT, Starke JR. Tuberculous osteomyelitis of the long bones in children. Pediatr Infect Dis J.1995; 14:524-6.

7. Jadhav RN, Palande DA. Calvarial tuberculosis. Neurosurgery 1999; 45:1345-50.

8. Garg RK. Tuberculosis of central nervous system. Postgrad Med J.1999; 75:133-40.

9. Vohra R, Kang HS, Dogra S, et al. Tuberculosis osteomyelitis. J Bone Joint Surg 1997; 79:562-6.

10. Bhandari B, Mandowara SL, Joshi H. Tubercular osteomyelitis of skull. Indian J Paediat.1981; 48:113-5.

11. Vallejo JG, Ong LT, Starke JR. Clinical features, diagnosis and treatment of tuberculosis in infants. Paediatrics. 1994; 941:1-7.

12. Degitz K. Detection of Mycobacterial DNA in the skin. Etiologic insights and diagnostic perspectives. Arch Dermatol.1996; 132:71-5.

13. Mohanty S, Rao CJ, Mukherjee KC. Tuberculosis of the skull. Int Surg 1981; 66:81-3. 